\title{
CAPITULOO 3
}

\section{AGUA DULCE: PERSPECTIVA DE UN SERVICIO ECOSISTÉMICO A PARTIR DE INDICADORES DE SOSTENIBILIDAD}

\author{
Carlos Mario Duque Chaves \\ Diego Alejandro Pérez Giraldo \\ Kevin Alberto Berthi Mantilla \\ Lina María Monsalve Castro \\ Víctor Fabián Forero Ausique \\ Diana Marcela Fúquene \\ Manuel Francisco Polanco Puerta ${ }^{5}$
}

\subsection{Introducción}

Las dinámicas de crecimiento poblacional, asociadas a la diversificación de sistemas productivos y las modificaciones antrópicas de la biocenosis, han conducido a un deterioro de los diversos ecosistemas y por consiguiente, una disminución de los diferentes servicios que ofrecen. El agua dulce, como servicio ecosistémico de abastecimiento, (conocido igualmente como suministro o aprovisionamiento), ha permitido a las sociedades evolucionar y crecer en torno a un recurso natural intrínsecamente ligado a su desarrollo, siendo un componente obligatorio para la producción de alimentos, energía, servicios y crucial en la supervivencia de los seres humanos.

La función de suministro de agua se refiere al filtrado, retención y almacenamiento de agua principalmente en arroyos, lagos y acuíferos, lo cual también depende del papel de los ecosistemas en los ciclos hidrológicos (de Groot et al., 2002), siendo utilizada en diversas actividades productivas, industriales, recreativas, sociales, entre otras. La gestión de este recurso se ha visto condicionada en diferentes aspectos, principalmente en el acceso y distribución a nivel mundial medida a partir de la disponibilidad, seguido por la afectación en términos de calidad. 
De acuerdo con el Programa Hidrológico Internacional adscrito a la UNESCO, los retos mundiales frente a la gestión de este recurso contempla en 2030 reducir el uso del agua tanto para el riego agrícola como la industria en un $20 \%$, y uso doméstico en un 15\%, al igual que el incremento de la productividad de agua en todos los sectores en un 50\%, acogiendo el enfoque de gestión de la demanda hídrica, el cual busca menos demanda de agua para los cultivos, mayor número de tecnologías de ahorro de agua y reutilización segura de aguas residuales.

El uso ineficiente y la escasez de agua, amenazan la futura producción de alimentos en el mundo y consecuentemente la seguridad alimentaria y el sustento de millones de productores y consumidores (IICA, 2017), lo cual ha quedado en manifiesto debido a los diferentes cambios ambientales característicos del antropoceno, que han llevado a definir acciones enfocadas en la adaptación más que en la prevención.

El sector académico cumple un papel importante en la implementación de los Objetivos de Desarrollo Sostenible relacionados con el agua. Abordar estos retos desde la ciencia requiere igualmente la articulación con el gobierno y la sociedad civil, por consiguiente, los aportes en términos de información, evaluación de fenómenos y proyecciones en diferentes horizontes, permiten aportar en la comprensión de recursos así como su dinámica en proyecciones futuras.

Fortalecer la gestión del agua constituye una necesidad para los gobiernos locales, regionales y sobre todo nacionales, lo cual ha posibilitado la implementación de instrumentos de gestión y planificación orientados, no solo a racionalizar el recurso, sino a brindar estrategias para su conservación. El presente capítulo se orienta principalmente en brindar una visión del agua dulce como servicio ecosistémico a partir de un enfoque de indicadores de sostenibilidad, profundizando principalmente en el agrícola debido a la presión que genera sobre los ecosistemas y requerimientos debido a su dinámica nacional.

\subsection{Agua dulce y agricultura}

En Colombia, al igual que en muchos países, el sector agropecuario se presenta como el principal usuario de agua en el mundo (IDEAM, 2015). Los requerimientos del recurso para suplir las necesidades de cultivos y procesos productivos asociados a estos, se encuentran sobre el $50 \%$ de la demanda total nacional. De acuerdo a las estimaciones realizadas para la formulación de la Política Nacional 
de Gestión Integral del Recurso Hídrico (PNGIHR), la demanda de agua por parte del sector agrícola en el año 2010 representó el 54\% del total de los recursos demandados a nivel nacional, estando por encima del uso doméstico, industrial, pecuario y de servicios.

En un estudio más reciente (IDEAM, 2015) la estimación del consumo de agua para este sector fue de 16.760 millones de metros cúbicos $\left(\mathrm{Mm}^{3}\right)$ encontrándose por debajo de las cifras presentadas en el año 2010. Las diferencias se fundamentan a partir de aspectos metodológicos e incertidumbres generadas entre otros aspectos, por la estimación de consumo en cultivos de pastos y de forraje de zonas geográficas como Orinoco y Amazonia. A pesar de lo anterior, es evidente el requerimiento predominante del sector agrícola frente a la utilización del recurso hídrico, por consiguiente, la presión ejercida puede suponer afectaciones sobre los recursos ecosistémicos, no solo asociados al agua, sino relacionados con coberturas boscosas, generación de residuos sólidos y residuos peligrosos, salud pública y socio-económicos.

A partir de los aportes realizados por Arévalo \& colaboradores (2014), se tiene que Colombia puede ofrecer la cantidad suficiente de agua para satisfacer los requerimientos de los sectores económicos, como lo son el agrícola, pecuario, doméstico, energético y minero-energético, así como de la población. Sin embargo, existen 22 subzonas hidrográficas llamadas "críticas" debido a la competencia entre los agricultores en unión con el sector pecuario y las áreas protegidas.

Las proyecciones de demanda hídrica total para Colombia al 2022, elaboradas en el marco del ENA por el MADS y el IDEAM, en el año 2014, ubican al sector agrícola con los requerimientos más altos en comparación con los demás sectores socioeconómicos, proyectándose un uso de agua total de $39.526 \mathrm{Mm}^{3}$, representando un incremento del $135,8 \%$ en comparación con la información disponible para año 2012. Igualmente, los resultado descritos en el ENA (estudio realizado cada cuatro años), en demanda anual ubica al área hidrográfica Magdalena - Cauca con un requerimiento de 20.247,23 $\mathrm{Mm}^{3}$ seguida del área Caribe $\left(4.904,88 \mathrm{Mm}^{3}\right)$, Orinoco (3.624,49 $\left.\mathrm{Mm}^{3}\right)$, Pacífico (1.281,22 $\mathrm{Mm}^{3}$ ) y Amazonas (162,93 $\mathrm{Mm}^{3}$ ). Las dinámicas de uso de agua para el sector agrícola son relativas a las características y condiciones naturales de cada área hidrográfica, es decir, si bien para Magdalena - Cauca la distribución porcentual para el uso agrícola es del 46,2\%, el área hidrográfica Caribe presenta un requerimiento superior con respecto a los demás usos, siendo este del 54,5\%. Para el área hidrográfica del Amazonas, el 
sector agrícola representa únicamente el 19,8\% de la distribución porcentual con respecto a otros usos, encontrándose por debajo del uso doméstico y pecuario.

En estudios realizados a nivel de cuencas hidrográficas como áreas donde confluyen diferentes aspectos ambientales, sociales, económicos y culturales, han permitido conocer la dinámica del recurso hídrico para los diferentes sistemas productivos allí presentes (Fonseca \& Cleves, 2018).

\subsection{Agua dulce y ganadería}

Los requerimientos de agua para el sector ganadero se encuentran relacionados no solo con la utilización del recurso para los animales en los diferentes ciclos de producción, sino también para garantizar el crecimiento de los forrajes requeridos para las dietas y producción de alimentos. En la búsqueda por minimizar los impactos ambientales generados por esta actividad productiva, la ganadería sostenible ha surgido como una herramienta para fortalecer, entre otros aspectos, el uso del recurso hídrico (Corredor, 2018). La apuesta de este enfoque de producción sostenible se orienta en garantizar los corredores ribereños con franjas de vegetación nativa que permita la protección de quebradas, lagos, humedales y arroyos.

De acuerdo con Ran \& colaboradores (2017), para prevenir el uso no sostenible y la gestión de los recursos hídricos, es necesario describir los vínculos entre la producción pecuaria y el uso de agua dulce. Lo anterior analizado desde un enfoque de indicadores de sostenibilidad, se relaciona principalmente con la identificación y cuantificación del recurso requerido en la cadena de producción, tanto utilizado directamente por los animales como de forma indirecta para aspectos de suministro de alimentos.

Bajo los criterios de la huella hídrica para el sector ganadero en Colombia, es necesario considerar que esta corresponde de manera indirecta a la alimentación del ganado, el cual consume pasto como fin económico asociado a la producción de leche o carne. Se estima en Colombia un requerimiento de agua para el sector pecuario de un total de $245.536 \mathrm{Mm}^{3} / a n ̃ o$, el cual se encuentra representado en pastos que sirven de alimento en la ganadería extensiva (sistemas silvopastoriles, pastos naturales y mejorados).

De acuerdo con los anteriores resultados, la presión sobre los recursos hídricos a partir de los requerimientos del sector ganadero debe ser evaluada desde su utilización en los diferentes ciclos de producción, así como los impactos ambientales 
derivados del desarrollo de la actividad, lo cual permita valorar de forma integral las afectaciones del sector.

\subsection{Indicadores de sostenibilidad hídrica}

Diferentes metodologías han surgido como herramienta para evaluar el desempeño en la gestión del recurso hídrico, así como la calidad del agua principalmente para consumo humano. A nivel nacional, se incorporó en el ENA 2014 la huella hídrica como instrumento de evaluación, la cual es aplicable a una unidad de análisis tan amplia como el territorio nacional, más específico como una cuenca hidrográfica o tan puntual como un producto o servicio.

Bajo un contexto nacional, la evaluación multisectorial de la Huela Hídrica $(\mathrm{HH})$ permitió incorporar una nueva herramienta de análisis con aplicación a nivel mundial, logrando presentar nuevos indicadores de acuerdo y conceptos poco utilizados en un estudio de orden nacional. La introducción de esta herramienta de análisis en el contexto académico hace parte de los trabajos realizados por Hoekstra \& Hung (2002), Chapagain \& Hoekstra (2003), Hoekstra (2003), Oki \& colaboradores (2003), Zimmer \& Renault (2003) y Fraiture \& colaboradores (2004). El marco de referencia para la aplicación de la $\mathrm{HH}$ se ha fortalecido en los últimos años, existiendo actualmente dos metodologías utilizadas para el cálculo (Vanham \& Bidoglio, 2013):

- El enfoque volumétrico de la Red de Huella Hídrica (WFN): Es el enfoque original desarrollado según Hoekstra \& Hung (2002) y se refiere a la cuantificación de los contenidos de agua requiriendo definir: A. Objetivos y alcance, B. Contabilidad, C. Evaluación de sostenibilidad, D. Respuestas.

- El enfoque de Análisis del Ciclo de Vida (LCA): Está de acuerdo con la reciente norma ISO 14046 y de manera similar a los estudios de ACV, debe llevarse a cabo de la siguiente manera: A. Definición de objetivo y alcance, $B$. Evaluación de inventario, C. Impacto, D. interpretación (Páez, 2018).

De acuerdo con el Centro de Análisis de Ciclo de Vida y Diseño Sustentable - CADIS, la Embajada de Suiza en Colombia y la Agencia Suiza para la Cooperación y el Desarrollo - COSUDE (2016), el enfoque presentado por la WFN busca cuantificar la cantidad de agua requerida por un consumidor, un producto, una empresa o una región geográfica divida en componentes azul, verde y gris. El enfoque de LCA busca cuantificar los impactos potenciales relacionados con el uso de agua en el ciclo de vida del producto, considerando impactos en el ecosistema, la salud humana y los recursos. 
En este sentido, el IICA (2017) define la evaluación de HH como una metodología que promueve y apoya el uso sostenible del recurso hídrico a través de información transparente y completa sobre el consumo y la contaminación del agua, en relación con la disponibilidad de esta. Lo anterior permite identificar de forma puntual el estado actual de procesos productivos, servicios, personas, áreas geográficas, hasta los requerimientos en términos del recurso para países.

En contexto nacional, se destacan estudios realizados por Arévalo \& colaboradores (2011), con aplicación al sector agrícola, WWF (2012), enfocado en la agricultura, CTA (2013), para la cuenca del río Porce, así como la introducción de la HH como herramienta de evaluación en el Estudio Nacional de Agua ENA elaborado en el año 2014. La articulación de esta herramienta se ha extrapolado a otros sectores como el energético, existiendo actualmente lineamientos puntuales para su aplicación en actividades productivas como la minería (UPME, 2016), en cuencas hidrográficas (CTA, 2013).

Adoptar nuevos enfoques para la evaluación del recurso hídrico a partir de indicadores de sostenibilidad ha permitido avanzar en la construcción de información orientada tanto a la calidad como en la cantidad (Fonseca, 2018). En este sentido, la aplicación de indicadores hídricos para evaluar el régimen natural y la intervención antrópica ha sido incluida en estudios de diferentes instituciones a nivel nacional, principalmente los desarrollados por el IDEAM. La tabla 3.1 relaciona los estudios desarrollados a nivel Latinoamericano para diferentes áreas de estudios, encontrándose elaborados principalmente para el campo agropecuario.

\subsection{Política de recurso hídrico}

La gestión orientada al recurso hídrico debe incluir lineamientos que integren diferentes componentes de índole no solo ambiental, sino social, económico y cultural. Bajo ese enfoque, el concepto de integralidad ha sido incluido como componente fundamental en los diferentes instrumentos de planificación que han sido definidos a nivel nacional.

De acuerdo con González (2017), el término “integrada” pasa a referirse a la gestión colectiva del recurso, ya que resalta la existencia de los diferentes valores que el agua representa para una multiplicidad de actores, llamando la atención sobre la importancia de definir espacios que promuevan la participación de diferentes actores que hacen parte de esta gestión. 
Tabla 3.1. Estudios de HH en Latinoamérica utilizando las metodologías Water Footprint Network - WFN y Life Cicle Assessment - LCA.

\begin{tabular}{|c|c|c|c|}
\hline Autor(es) & País & Área de estudio & Metodología* \\
\hline Arena et al., (2011) & Argentina & Soya & WFN \\
\hline Valenzuela (2011) & Chile & Producción de cobre & WFN \\
\hline Arévalo et al., (2011) & Colombia & Producción agrícola nacional & WFN \\
\hline Güereca et al., (2011) & $\begin{array}{l}\text { Latinoamérica } \\
\text { y el Caribe }\end{array}$ & $\begin{array}{l}\text { Tratamiento de aguas } \\
\text { residuales }\end{array}$ & LCA \\
\hline Farell et al., (2011) & México & Trigo & LCA \\
\hline Arévalo (2012) & Colombia & $\begin{array}{l}\text { Productos agrícolas } \\
\text { relevantes }\end{array}$ & WFN \\
\hline Vásquez y Buenfil (2012) & Latinoamérica & $\begin{array}{l}\text { Diferentes sectores } \\
\text { socioeconómicos }\end{array}$ & WFN \\
\hline Franke y Castro (2012) & Brasil & Productos cosméticos & WFN \\
\hline Farell et al., (2013) & México & Agua de uso público & LCA \\
\hline Farell (2013) & México & $\begin{array}{l}\text { Diferentes sectores } \\
\text { socioeconómicos }\end{array}$ & LCA \\
\hline $\begin{array}{l}\text { CTA, SuizAgua Colombia } \\
\text { y Cosude (2013) }\end{array}$ & Colombia & Cuencas hídricas & WFN \\
\hline $\begin{array}{l}\text { Gobierno de la provincia } \\
\text { de San Luis (2013) }\end{array}$ & Argentina & Agrícola y pecuaria & WFN \\
\hline Ran et al., (2013) & Uruguay & Ganadería & WFN \\
\hline Peña et al., (2013) & Chile & Producción de cobre & WFN \\
\hline Vanegas et al., (2013) & Colombia & Producción de Lirio Japonés & WFN \\
\hline $\begin{array}{l}\text { Servicios Ambientales S.A. } \\
-(2013)\end{array}$ & Quito & Embotellado de bebidas & WFN \\
\hline Fernanda França Ferreira (2014) & Brasil & Energía eléctrica & WFN \\
\hline $\begin{array}{l}\text { Ximena Vanessa Echeverri } \\
\text { Bedoya (2014) }\end{array}$ & Colombia & Piedra caliza & WFN \\
\hline Sánchez., et al., (2014) & Colombia & Pitahaya Amarilla & WFN \\
\hline Moyano Salcedo et al.,(2015) & Argentina & Leche & WFN \\
\hline $\begin{array}{l}\text { Instituto Nacional de } \\
\text { Tecnología Agropecuaria - INTA } \\
\text { (2015) }\end{array}$ & Argentina & Leche & WFN \\
\hline $\begin{array}{l}\text { Instituto Nacional de } \\
\text { Tecnología Agropecuaria - INTA } \\
\text { (2015) }\end{array}$ & Argentina & Maní & WFN \\
\hline $\begin{array}{l}\text { Universidad de Pamplona. } \\
\text { GAIA Servicios Ambientales } \\
\text { (2015) }\end{array}$ & Colombia & Cacao & WFN \\
\hline Díaz et al., (2016) & Colombia & Algodón & WFN \\
\hline
\end{tabular}

Fuente: autor a partir de CADIS (2016). 
Para el caso colombiano, la PNGIHR tiene una meta a 12 años, en los cuales se desarrollarán las líneas de acción estratégicas de acuerdo con lo planteado a corto (2014), mediano (2018) y largo plazo (2022), a través del Plan Hídrico Nacional. Bajo el objetivo general (MAVDT 2010), el MAVDT plantea diferentes objetivos desde diferentes escenarios de gestión, definiendo acciones puntuales para aspectos como la oferta, demanda, calidad, riesgo, fortalecimiento institucional y gobernabilidad. Puntualmente, los objetivos definidos en el PNGIRH se orientan en:

a. Objetivo 1. OFERTA: Conservar los ecosistemas y los procesos hidrológicos de los que depende la oferta de agua para el país.

b. Objetivo 2. DEMANDA: Caracterizar, cuantificar y optimizar la demanda de agua en el país.

c. Objetivo 3. CALIDAD: Mejorar la calidad y minimizar la contaminación del recurso hídrico.

d. Objetivo 4. RIESGO: Desarrollar la gestión integral de los riesgos asociados a la oferta y disponibilidad del agua.

e. Objetivo 5. FORTALECIMIENTO INSTITUCIONAL: Generar las condiciones para el fortalecimiento institucional en la gestión integral del recurso hídrico.

f. Objetivo 6. GOBERNABILIDAD: Consolidar y fortalecer la gobernabilidad para la gestión integral del recurso hídrico.

Un aspecto importante contenido en esta política se relaciona con el componente inmerso en los cuatro primeros objetivos y que se orienta en garantizar la planificación del recurso a partir de los diferentes componentes de los ecosistemas, tales como suelo, fauna, flora, consumo y uso del agua. Bajo los objetivos 5 y 6 , se orienta un componente de planeación a partir del fortalecimiento de las capacidades institucionales y principalmente la gobernabilidad.

Posterior a la formulación e implementación de la PNGIHR, se han definido otros instrumentos en la búsqueda de garantizar el cumplimiento de los objetivos propuestos. Uno de los más importantes se ha relacionado con los Planes de Ordenación y Manejo de Cuencas Hidrográficas (POMCA) con aplicación en las subzonas hidrográficas, el cual define criterios y lineamientos puntuales para ser implementados de acuerdo a las dinámicas del recurso hídrico. 


\subsection{Consideraciones finales}

Es evidente que la gestión de los recursos hídricos requiere soluciones puntuales y dinámicas, que articulen nuevos enfoques y que permitan abordar los desafíos que conlleva la seguridad hídrica producto del incremento de la población y de los sistemas productivos, así como los cambios en la sociedad derivados de la adaptación al cambio climático. Bajo esta perspectiva, soluciones basadas en conceptos biomiméticos como propuesta para afrontar los retos tanto de disponibilidad, calidad y riesgos relacionados con el agua, surgen como una alternativa en la búsqueda de alcanzar el Objetivo de Desarrollo Sostenible ODS 6, en relación a la gestión sostenible de estos recursos, así como el saneamiento.

El programa de la WWAP (2018), adscrito a ONU-Agua presenta en el Informe Mundial sobre el desarrollo de los recursos hídricos una nueva propuesta de soluciones basadas en la naturaleza ( $\mathrm{SbN}$ ), las cuales imitan los procesos naturales para contribuir a la gestión mejorada del agua.

Los retos que trae consigo la adaptación al cambio climático conllevan a implementar acciones desde diferentes enfoques, en caso puntual de la gestión hídrica, nuevos paradigmas han comenzado a orientar el énfasis dejando a un lado la observación de los impactos en los ecosistemas y coincidiendo con la gestión de los mismos ecosistemas con el fin de alcanzar los beneficios ambientales.

Estrategias como la implementación de las SbN a escala de cuencas hidrográficas y estrategias como el Pago por Servicios Ambientales - PSA mediante incentivos, asistencias y materiales, permiten a las comunidades ubicadas tanto aguas arriba como aguas abajo, poder obtener beneficios como purificación del agua, reducción en los riesgos de inundación y reducción de la erosión a raíz de la no ampliación de la frontera agrícola. Acciones puntuales de la SbN como gestión de cuencas hidrográficas, agricultura de conservación, infraestructura verde urbana, humedales construidos, protección y restauración de ecosistemas, entre otros, posibilitan alcanzar las metas del ODS 6 en relación al agua y saneamiento.

Finalmente, avanzar en la construcción de una gestión orientada en la resiliencia del agua a través del Antropoceno requiere re-definir la relación entre los ecosistemas, la hidrogeología y las necesidades humanas, en este sentido, los instrumentos de planifcación que se articulen a los ODS, deben partir de la integración de estrategias y nuevos enfoques que puedan posicionar al agua como eje articulador del Desarrollo Sostenible. 


\subsection{Estudio de caso: Cuenca del Río Otún}

La cuenca hidrográfica como espacio de interacción socioecológica, en la cual confluyen una diversidad de actores, sirve como soporte para las relaciones entre el aprovisionamiento de los servicios ecosistémicos y las interacciones sociales, propias de los procesos de desarrollo que dan lugar a los diferentes cambios en las coberturas y usos del suelo. Estas dinámicas requieren a corto y mediano plazo acciones puntuales de planificación, orientadas no solo a la gestión de los recursos hídricos, sino a garantizar un equilibrio entre el componente social y económico, representado en el aprovechamiento de los recursos naturales existentes en estos territorios.

El río Otún constituye la principal fuente abastecedora de agua para la ciudad de Pereira (Risaralda) y es un territorio estratégico por los diferentes servicios ecosistémicos que allí se desarrollan. Las dinámicas naturales, económicas y sociales presentes en la cuenca media y alta del río Otún, han generado el desarrollo de proyectos de investigación orientados a fortalecer la Gestión Integral de Recurso Hídrico -GIRH, así como procesos enfocados a su planificación mediante la formulación del Plan de Ordenamiento y Manejo de Cuencas Hidrográficas, el cual se encuentra en desarrollo.

De acuerdo con CTA \& colaboradores (2015), la subzona hidrográfica perteneciente al río Otún y otros directos al Cauca (área hidrográfica Magdalena-Cauca) presenta un Índice de Presión Hídrica a los Ecosistemas -IPHE ${ }^{6}$ en una categoría "critica" (Tabla 3.2), siendo una cuenca con competencia entre el sector agropecuario y los ecosistemas estratégicos para la provisión de servicios ecosistémicos, principalmente asociado a la ampliación de la frontera agrícola.

De acuerdo con los resultados de la Evaluación Multisectorial de la Huella Hídrica en Colombia (CTA et al., 2015), es necesario continuar con los procesos de aplicación de las áreas protegidas, fortalecer los procesos de planeación de actividades productivas como la expansión ganadera y agrícola, así como la articulación con las estrategias de adaptación al cambio climático que se implementen tanto a nivel regional como nacional. 
Tabla 3.2. Subzonas hidrográficas con IPHE crítico.

\begin{tabular}{l|l|l|c}
\multicolumn{1}{c|}{ SZH } & \multicolumn{1}{|c|}{ Subzona hidrográfica } & \multicolumn{1}{|c}{ Área hidrográfica } & IPHE \\
\hline 3501 & Río Metica (Guamal-Humadea) & Orinoco (3) & 1,77 \\
\hline 4505 & Río Luisa & Amazonas (4) & 1,47 \\
\hline 2634 & Río Cali & Magdalena Cauca (2) & 1,44 \\
\hline 2405 & Río Sogamoso & Magdalena Cauca (2) & 1,34 \\
\hline 2314 & Río Opón & Magdalena Cauca (2) & 1,26 \\
\hline 2903 & Canal del Dique margen derecho & Magdalena Cauca (2) & 1,22 \\
\hline 1204 & $\begin{array}{l}\text { Río Canalete y otros Arroyos Directos } \\
\text { al Caribe }\end{array}$ & Caribe (1) & 1,21 \\
\hline 2613 & Río Otún y otros directos al Cauca & Magdalena Cauca (2) & 1,21 \\
\hline 2303 & $\begin{array}{l}\text { Directos al Magdalena entre ríos } \\
\text { Seco y Negro (md) }\end{array}$ & Magdalena Cauca (2) & 1,20 \\
\hline 130 & Bajo Sinú & Caribe (1) & 1,19 \\
\hline
\end{tabular}

Fuente: CTA et al., (2015).

\subsection{Evaluación del capítulo}

1. ¿La aplicación de la Huella Hídrica como herramienta de análisis permite evaluar la sostenibilidad en diferentes sistemas de producción?

2. ¿Cuáles son los criterios conceptuales necesarios para proponer la evaluación del recurso hídrico mediante indicadores de sostenibilidad?

3. ¿Cuáles son las estrategias que se pueden aplicar a nivel de cuenca hidrográfica que puedan permitir la disminución del Índice de Presión Hídrica a los Ecosistemas IPHE?

\section{Referencias}

Arévalo, D., Lozano, J., \& Sabogal, J. (2014). Estudio nacional de huella hídrica Colombia sector agrícola. Revista Internacional de Sostenibilidad, Tecnología y Humanismo, 6, 101-126.

CADIS, Embajada de Suiza en Colombia, COSUDE. (2016). Huella de Agua (ISO 14046) en América Latina, análisis y recomendaciones para una coherencia regional. Centro de Análisis de Ciclo de Vida y Diseño Sustentable - Agencia Suiza para la Cooperación y el Desarrollo.

Corredor, E. S. (2018). Huella hídrica. En Fonseca, J. A., Páez, E.M. y Corredor, E. S. Metodologías para la estimación de sostenibilidad agropecuaria. (pp: 93-114). Colombia. Sello editorial UNAD 
CTA. (2013). Evaluación de la Huella Hídrica en la cuenca del río Ponce. Medellín: Centro de Ciencia y Tecnología de Antioquia.

Chapagain, A.K., \& Hoekstra, A.Y. (2003). Virtual water trade. Virtual water flows between nations in relation to trade in livestock and livestock products. In: Hoekstra, A.Y. (Ed). Virtual Water Trade Proceedings of the International Expert Meeting on Virtual Water Trade (pp. 49-76). IHE Delft, The Netherlands: Research Report Series No. 12.

CTA, GSI-LAC, COSUDE, IDEAM, (2015). Evaluación Multisectorial de la Huella Hídrica en Colombia. Resultados por subzonas hidrográficas en el marco del Estudio Nacional del Agua 2014. Medellín, Colombia: Centro de Ciencia y Tecnología de Antioquia, GSI-LAC, Agencia Suiza para la Cooperación y el Desarrollo, Instituto de Hidrología, Meteorología y Estudios Ambientales de Colombia.

De Fraiture, C., Cai, X., Amarasinghe, U., Rosegrant, M., \& Molden, D. (2004). Does international cereal trade savewater? The impact of virtual water trade on global water use. Comprehensive Assessment Research Report, vol. 4.

De Groot, R.S., Wilson, M.A., \& Boumans, R.M.J. (2002). A typology for the classification, description and valuation of ecosystem functions, goods and services. Ecological Economics, 41(3), 393-408.

Fonseca, J.A. (2018). Evaluación de Agroecosistemas Familiares Campesinos Mediante Indicadores de Sustentabilidad. En Fonseca, J. A., Páez, E.M. y Corredor, E. S. Metodologías para la estimación de sostenibilidad agropecuaria. (pp: 93-114). Colombia. Sello editorial UNAD.

Fonseca, J.A. y Cleves, J.A. (2018). Evaluación de la sustentabilidad de agroecosistemas familiares campesinos en zonas de páramo en Colombia. En Astier, M y Arnes, E.(Ed.). (2018). Sostenibilidad en sistemas de manejo de recursos naturales en países andinos. (pp; 79 - 122). Ciudad de México. México. Editorial libros UNAM.

González, N. (2017). Desafíos de la gobernanza ambiental: una aproximación a las implicaciones de la Gestión Integrada del Recurso Hídrico en Colombia. Ciencia Política, 12(23), 205-229.

Hoekstra, A.Y. (2003). Virtual water trade. Proceedings of the international expert meeting on virtual water trade. In: Hoekstra, A.Y. (Ed). Virtual Water Trade Proceedings of the International Expert Meeting on Virtual Water Trade (pp. 13-23). IHE Delft, The Netherlands: Research Report Series No. 12.

Hoekstra, A.Y., \& Hung, P.Q. (2002). Virtual water trade. A quantification of virtual water flows between nations in relation to international trade. In: Hoekstra, A.Y. (Ed). Virtual Water Trade Proceedings of the International Expert Meeting on Virtual Water Trade (pp. 25-47). IHE Delft, The Netherlands: Research Report Series No. 12. IDEAM. (2015). Estudio Nacional del Agua 2014. Bogotá., D. C., Colombia: Instituto de Hidrología, Meteorología y Estudios Ambientales.

IICA. (2017) Guía metodológica para la evaluación de la huella hídrica en una cuenca hidrográfica. Unión Europea - San José, C.R.: Instituto Interamericano de Cooperación para la Agricultura.

MAVDT. (2010). Política Nacional para la Gestión Integral del Recurso Hídrico. Bogotá, D.C.: Colombia, Ministerio de Ambiente, Vivienda y Desarrollo Territorial.

Oki, T., Sato, M., \& Kawamura, A. (2003). Virtual water trade to Japan and in the world. In: A.Y. Hoekstra (Ed). Virtual Water Trade Proceedings of the International Expert Meeting on Virtual Water Trade (pp. 221-235). IHE Delft, The Netherlands: Research Report Series No. 12. 
Páez, E. M. (2018). Huella de carbono. En Fonseca, J. A., Páez, E.M. y Corredor, E. S. Metodologías para la estimación de sostenibilidad agropecuaria. (pp: 48-92). Colombia. Sello editorial UNAD.

Ran, Y., van Middelaar, C.E., Lannerstad, M., Herrero, M., \& de Boer, I.J.M. (2017). Freshwater use in livestock production-to be used for food crops or livestock feed? Agricultural Systems, 155, 1-8.

UNESCO. (2014). International Hydrological Programme (2014) Water in the Post-2015 Development Agenda and Sustainable Development Goal. Paris, France: United Nations Educational Scientific and Cultural Organization.

UPME. (2016). Guía metodológica para evaluación de la Huella Hídrica del sector minero Colombiano. Bogotá D. C., Colombia: Unidad de Planeación Minero Energética.

Vanham, D., \& Bidoglio, G. (2013). A review on the indicator water footprint for the EU28. Ecological Indicators, 26, 61-75.

WWF. (2012). Una mirada a la agricultura de Colombia desde su Huella Hídrica. Bogotá D. C., Colombia: Reporte Colombia World Wildlife Fund. Recuperado de: http://www.huellahidrica. org/Reports/Arevalo-2012-HuellaHidricaColombia.pdf

WWAP/ONU-Agua (2018). Informe Mundial de las Naciones Unidas sobre el Desarrollo de los Recursos Hídricos: Soluciones basadas en la naturaleza para la gestión del agua. París, Francia: UNESCO. http://unesdoc.unesco.org/images/0026/002614/261494s.pdf

Zimmer, D., Renault, D. (2003). Virtual water in food production and global trade: review of methodological issues and preliminary results. In: A.Y. Hoekstra (Ed). Virtual Water Trade Proceedings of the International Expert Meeting on Virtual Water Trade (pp. 93-109). IHE Delft, The Netherlands: Research Report Series No. 12. 